(3,500 m. above sea-level) without absorber and under $2 \mathrm{~cm}$. lead respectively. For the correct evaluation of the transition effect the figures of the second column should be multiplied by the absorption factor for $2 \mathrm{~cm}$. lead of the star-producing radiation, which is easily deduced from the mean path in lead of the same radiation. We then obtain, using the mean path of $29 \mathrm{~cm}$. of lead from George and Jason ${ }^{2}$, the figures of the fourth column in Table 1.

As we have not yet obtained data for materials other than lead, the effect is insufficient in itself to give us an idea about the secondary radiation responsible for it. But from many different considerations, which will be discussed in detail elsewhere, it seems reasonable to consider that this secondary radiation is generally composed of nucleons produced in the nuclear evaporations themselves.

With convenient mean angular distribution and mean multiplicity of the stars, it is possible to explain the rather small thickness where the maximum occurs ; and at the same time, the rather low energy of the nucleons gives a reason for the difference of behaviour between stars with few prongs and those with many.

Table 2. Total numbers of stars found in the same Ilford $C 2$ plate, 1 in. $\times 2$ in., 100 microns thick, with two different scanning-rates

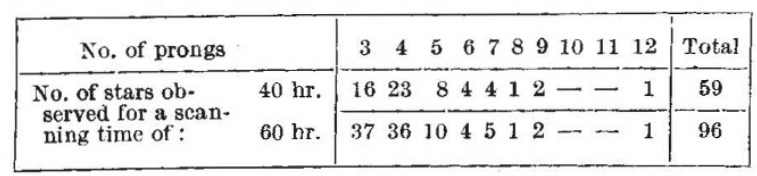

To explain the difference between our results and the results of other authors, we must consider the following. First, the three-prong stars, which are responsible for the transition effect, fade much more easily than stars with many prongs; and we can say, from some orientation measurements, that this fading is not negligible and is strongly variable in different makes of the same type of emulsion. Secondly, the three-prong stars are easily missed as a consequence of any slight inconvenience in the scanning or developing. We purposely tested this, and we found that, on trying to speed up slightly the scanning time, there was a sensible diminution in the number of four-prong stars, and a great decrease in the number of three-prong stars, so that the graph of frequency of stars plotted against number of prongs showed a maximum for the four-prong stars. In Table 2 , the figures are given for an Ilford $C 2$ plate, 1 in. $\times 2$ in., 100 microns thick, scanned twice at two different rates, corresponding to a time of about $40 \mathrm{hr}$. and $60 \mathrm{hr}$. respectively for the scanning of the whole plate. Moreover, it is constantly our experience that the check with high magnification (oil objective, $\times 100$ ) of every star considerably affects the shape of the diagram of the frequency of the stars against number of prongs.

We are grateful to Prof. G. Bernardini for his help, and to Prof. E. Amaldi for a full discussion of the data.

Centro di Fisica Nucleare,

G. Cortini

A. Manfredini

Istituto di Fisica dell'Università,

Rome.

April 5.

1 Bernardini, G., Cortini, G., and Manfredini, A., Nuovo Cimento, 5, 511 (1948); Phys. Rev., 74, 845 (1948).

seorge, E. P., and Jason, A. C., private communieation.

s Private communication.

\section{Hyperfine Structure in the Solid State}

A HYPERFINE structure has been observed in the microwave resonance-absorption band of a dilute Tutton salt, in which 95 per cent of the copper had been replaced by magnesium.

The resonance in this salt was investigated at a wave-length of $3.2 \mathrm{~cm}$. in a magnetic field of about 3,000 oersted. The resonance band (due to reversal of the electronic spin in the magnetic field) was resolved into four lines, the distances between adjacent lines being about 93 oersted, when the magnetic field was directed along the $K_{1}$-axis. Evidently the applied field is sufficient to decouple the electronic from the nuclear spin, the four lines corresponding to the four possible orientations of the copper nucleus $(I=3 / 2)$ in the external field.

The two copper isotopes 63 and 65 have somewhat different magnetic moments $^{1}$; but the corresponding sets of lines were not resolved.

lieasurements were made with the magnetic field in various orientations in the a.c. plane. The overall structure varied by a factor of about $1 \cdot 5$, being a maximum along the $K_{1}$-axis, where the electronic $g$ is a maximum, and a minimum near the $K_{2}$-axis, where the electronic $g$ is a minimum.

Some time ago, L. J. F. Broer carried out calcula. tions on the basis of Polder's ${ }^{2}$ theory of the magnitude of the hyperfine structure in copper salts. The in. fluence of hyperfine structure ${ }^{3}$ had also been found experimentally by F. W. de Vrijer in the specific heat of the spin system of the same diluted copper salt. The absence of any observable hyperfine strueture in the undiluted salt may be explained by the influence of magnetic interaction and exchange interaction between the copper ions. However, it is probable that the hyperfine structure contributes to the band-widths in copper salts ${ }^{4}$.

\section{R. P. Penrose}

THE discovery described above was made by Dr. Penrose in the autumn of 1948. After his tragic death on April 28, 1949, I wrote this note, making use of a draft written by Dr. Penrose while in hospital.

Kamerlingh Onnes Laboratorium, C. J. GorTER Leyden. May 12.

${ }^{1}$ Pound, R. V., Phys. Rev., 78, 745 (1947).

a Polder, D., Physica, 9, 709 (1942).

sorter, C. J., Physica, 14, 504 (1948).

+ Bagguley, D. M. S., Bleaney, B., Griffiths, J. H. E., Penrose, R. P. and Plunipton, B. I., Proc. Phys. Soc., 61. 542 and 551 (1948). Bagguley. 1). M. S., Griffiths, J. H. E., and Pryce. M. H. I. Nature, 168, 538 (1948).

WE had the privilege of hearing of his results from Dr. Penrose shortly after his discovery of the hyperfine structure of paramagnetic resonance, and of discussing them with him when he was on a visit to Oxford. We were stimulated to make some calculations on the effect, in particular its variation with orientation of the applied field, and kept up a correspondence with him on this subject, which was interrupted by his death. The variation observed by Penrose differs markedly from the expected variation calculated by us on the basis of Polder's theory, and we believe that the discrepancy indicates an influence of the crystalline electric field on paramagnetic ions more complicated than has been generally assumed. 